\title{
In vitro polymyxin activity against clinical multidrug-resistant fungi
}

Hanane Yousfi', Stéphane Ranque ${ }^{2}$, Jean-Marc Rolain ${ }^{1}$ and Fadi Bittar ${ }^{1 *}$ (D)

\begin{abstract}
Background: Although antifungals are available and usually used against fungal infections, multidrug-resistant (MDR) fungal pathogens are a growing problem for public health. Moreover, fungal infections have become more prevalent nowadays due to the increasing number of people living with immunodeficiency. Thus, previously rarelyisolated and/or unidentified fungal species including MDR yeast and moulds have emerged around the world. Recent works indicate that polymyxin antibiotics (polymyxin B and colistin) have potential antifungal proprieties. Therefore, investigating the in vitro activity of these molecules against clinical multidrug-resistant yeast and moulds could be very useful.

Methods: In this study, a total of 11 MDR yeast and filamentous fungal strains commonly reported in clinical settings were tested against polymyxin antibiotics. These include strains belonging to the Candida, Cryptococcus and Rhodotorula yeast genera, along with others belonging to the Aspergillus, Fusarium, Scedosporium, Lichtheimia and Rhizopus mould genera. The fungicidal or fungistatic action of colistin against clinical yeast strains was determined by the time-kill study. Further, a checkerboard assay for its combination with antifungal agents, usually used in clinical practices (amphotericin B, itraconazole, voriconazole), was carried out against multi-drug resistant fungal strains.

Results: Polymyxin B and colistin exhibited an antifungal activity against all MDR fungal strains tested with MICs ranging from 16 to $128 \mu \mathrm{g} / \mathrm{ml}$, except for the Aspergillus species. In addition, colistin has a fungicidal action against yeast species, with minimum fungicidal concentrations ranging from 2 to 4 times MICs. It induces damage to the MDR Candida albicans membrane. A synergistic activity of colistin-amphotericin B and colistin-itraconazole associations against Candida albicans and Lichtheimia corymbifera strains, respectively, and colistin-fluconazole association against Rhodotorula mucilaginosa, was demonstrated using a checkerboard microdilution assay.
\end{abstract}

Conclusion: colistin could be proposed, in clinical practice, in association with other antifungals, to treat lifethreatening fungal infections caused by MDR yeasts or moulds.

Keywords: Polymyxin antibiotics, MDR-fungi, Repurposing-drug, Candida albicans, Molds

\section{Background}

Invasive fungal diseases treatment is challenged by the restricted number of available antifungal drugs; with only four different classes of antifungals being available to treat a large number of fungal-associated diseases [1]. (i) Polyenes are the first antifungals available in clinical practice with two drugs mainly used: amphotericin B and nystatin. (ii) Azole antifungals, such as fluconazole, itraconazole, voriconazole, posaconazole and isavuconazole, are the

\footnotetext{
* Correspondence: fadi.bittar@univ-amu.fr

${ }^{1}$ Aix Marseille Univ, IRD, APHM, MEPHI, IHU Méditerranée Infection, 19-21

boulevard Jean Moulin, 13005 Marseille, France

Full list of author information is available at the end of the article
}

most common drugs used in different clinical situations; azole drugs display a large spectrum of activity against both yeast and filamentous fungi. (iii) Pyrimidine analogues, including 5-flucytosine, are used in combination with other antifungals to treat yeast infections but have little action against most moulds. (iv) Finally, echinocandins, the newest class of antifungals include caspofungin, micafungin and anidulafungin that display fungicidal activity against ascomycetes yeast species [2].

In addition to this limited therapeutic arsenal, there has been a dramatic and worldwide increase in the incidence of fungal infections [3]. In fact, along with the

(c) The Author(s). 2019 Open Access This article is distributed under the terms of the Creative Commons Attribution 4.0 International License (http://creativecommons.org/licenses/by/4.0/), which permits unrestricted use, distribution, and reproduction in any medium, provided you give appropriate credit to the original author(s) and the source, provide a link to the Creative Commons license, and indicate if changes were made. The Creative Commons Public Domain Dedication waiver (http://creativecommons.org/publicdomain/zero/1.0/) applies to the data made available in this article, unless otherwise stated. 
main mycosis agents, such as Candida albicans, Aspergillus fumigatus and Cryptococcus neoformans [4], other life-threatening and emerging pathogens, including not previously well-identified/characterized species and opportunistic multidrug-resistant (MDR) ones, are increasingly reported. These include Candida auris, Scedosporium/Lomentospora spp., Fusarium spp. and Mucorales [2]. Indeed, several factors can explain this increasing incidence of fungal infections; the increasing number of patients with immunodeficiency (ex. HIV, cancer and transplant patients), the ageing of the population [5] and improved detection and diagnostic methods [2]. However, the severity of such fungal infections varies depending on the site of infection (superficial or deep-seated) and the immune status of the concerned patients. One major characteristic of these emerging fungal pathogens is their highly-resistant profile to antifungal drugs. Therefore, these disseminated infections caused by MDR yeasts and moulds are difficult to treat [6], leading thus to a significant increase of morbidity and mortality, in immunocompromised patients but also in healthy individuals [7]. Antifungal resistance can be intrinsic, called primary resistance, or acquired, also called secondary resistance. Many resistance mechanisms have been described, such as biofilm formation (especially in Candida albicans), failure of intracellular drug accumulation or drug target alterations [8]. During the past decade, genome plasticity of human fungal pathogens has been strongly associated with their ability to acquire resistance to antifungals [9]. That is why many studies suggest to use other pharmacological classes and re-purposing old drugs either as a single antifungal agent or in combination with known antifungal drugs [10].

In this regard, antimicrobial peptides (AMPs) have received attention as prospective compounds for a further discovery of new antimycotics. More than 2700 antimicrobial peptides have been identified and the number is growing [11]. Among the AMPs commonly used in therapeutic practices, there are polymyxins which are cyclic, positively charged peptides, obtained naturally from Gram-positive bacteria, such as Paenibacillus polymyxa. Among polymyxin molecules described, two have been used in clinical settings: polymyxin B (PMB) and polymyxin E (colistin) [12]. This class of antibiotics has been discarded in the early 1980s because of their neuro- and nephro-toxicity. However, polymyxins were recently reintroduced in the antimicrobial therapy as a last option to treat infections caused by multidrug-resistant Gram-negative bacteria [13]. In addition to its antibacterial action, polymyxins were shown in the early 1970s to have antifungal activity against various Candida species, including Candida tropicalis with polymyxin E Minimum inhibitory concentrations (MICs) ranging from 30 to $75 \mu \mathrm{g} / \mathrm{ml}$ [14]. More recently, polymyxin sensitivity of life-threatening moulds, such as Fusarium and Rhizopus species, has been described $[15,16]$. This study aimed to test the in vitro activity of polymyxin against the most common clinical MDR yeasts and moulds and to assess colistin activity, the mechanism of action and the synergy of colistin-antifungals associations that could be used in the treatment of invasive fungal infections.

\section{Methods \\ Fungal isolates}

In this study, eleven clinical fungi recovered at the University hospital of Marseille were used. Four yeasts belonging to Candida, Cryptococcus and Rhodotorula species and seven moulds belonging to Fusarium, Scedosporium, Lichtheimia, Rhizopus and Aspergillus species were tested (Table 1). Isolates were identified using Matrix-Assisted Laser Desorption/Ionisation mass spectrometry (MALDI-TOF MS) [17] and microscopic methods for the filamentous fungi species. These strains were isolated from different clinical samples including blood culture, cerebrospinal fluid, nails, bronchial aspiration and ocular samples (Table 1). Candida krusei ATCC 6258, Candida parapsilosis ATCC 22019, Aspergillus fumigatus ATCC 204205, Aspergillus flavus ATCC 204304, Escherichia coli LH1 [18], Escherichia coli 1R4 [19] and Klebsiella pneumoniae 853 [20] were used as susceptibility testing quality controls.

\section{Phenotypic profiles determination}

Antifungal susceptibility testing was performed using two different methods: E-test (BioMérieux, Marcy l'Etoile, France) and commercial broth microdilution plates; Sensititre ${ }^{\circ}$ YeastOne ${ }^{\bullet}$ (Thermo Fisher Scientific, Schwerte, Germany). The MICs obtained for each antifungal tested against yeasts and moulds (Table 1) was compared to the breakpoints provided by the manufacturers or to the epidemiological cutoff as previously described [21] in order to assess the susceptibility of each strain to the different antifungal agents tested.

\section{Polymyxin susceptibility testing}

Colistin and PMB MICs were performed using the broth microdilution method as outlined by the Clinical and Laboratory Standards Institute (CLSI) (M38-A, Vol. 22, NO. 16 for filamentous fungi and M27-A2, Vol. 22, NO. 15 for yeasts). Serial colistin and PMB (Sigma Aldrich, St Louis, France) dilutions ranging from 0.5 to $256 \mu \mathrm{g} /$ $\mathrm{ml}$ were prepared in RPMI-1640 (Sigma Aldrich, St Louis, France) with glutamine and without bicarbonate medium buffered to pH 7.0 with MOPS (Sigma Aldrich, St Louis, France) buffer. Fungal inoculums were prepared in the test medium and adjusted to 0.5 
Table 1 Phenotypic profiles and Colistin, PMB MICs of fungal strains tested in this study

\begin{tabular}{|c|c|c|c|c|c|c|c|c|c|c|c|c|c|}
\hline & \multirow[t]{2}{*}{ Clinical samples } & \multicolumn{12}{|c|}{ MICs $(\mu \mathrm{g} / \mathrm{mL})$} \\
\hline & & Anid & Mica & Caspo & Flu & $5-\mathrm{Fc}$ & Posa & Itra & Vorico & $A B$ & Isavu & $\mathrm{Ct}$ & PMB \\
\hline Candida krusei ATCC 6258 & / & 0.06 & 0.12 & 0.25 & 32 & 8 & 0.25 & 0.12 & 0.25 & 1 & ND & 64 & 32 \\
\hline Candida parapsilosis ATCC 22019 & / & 0.5 & 0.5 & 0.12 & 1 & 0.12 & 0.03 & 0.008 & 0.03 & 0.5 & ND & 64 & 16 \\
\hline Rhodotorula mucilaginosa & Endobucal & $>8$ & $>8$ & $>8$ & 128 & 0.06 & 1 & 0.5 & 2 & 1 & ND & 32 & 16 \\
\hline Cryptococcus neoformans & Blood & $>8$ & $>8$ & $>8$ & 2 & 1 & 0.03 & 0.12 & 0.03 & 0.5 & ND & 32 & 16 \\
\hline Candida albicans $\mathrm{H} 5$ & CSF & 0.015 & 0.03 & 0.05 & 16 & 1 & 0.5 & 0.5 & 0.25 & 0.25 & ND & 128 & 128 \\
\hline Candida albicans $\mathrm{H} 6$ & Nails & 0.06 & 0.03 & 0.12 & $>256$ & 0.12 & $>8$ & 32 & 256 & 1 & ND & 128 & 64 \\
\hline Aspergillus fumigatus ATCC 204205 & / & ND & ND & ND & ND & ND & 0.008 & 0.015 & 0.12 & 2 & ND & $>256$ & $>256$ \\
\hline Aspergillus flavus ATCC 204304 & / & ND & ND & ND & ND & ND & 0.06 & 0.06 & 1 & 4 & ND & $>256$ & $>256$ \\
\hline Aspergillus calidoustus & Bronchial aspiration & ND & ND & ND & ND & ND & 1 & 6 & 4 & 4 & 0.25 & 256 & 128 \\
\hline Fusarium oxysporumY5 & Nails & ND & ND & ND & ND & ND & $>32$ & $>32$ & 2 & 4 & $>32$ & 64 & 16 \\
\hline Fusarium solani Y6 & Ocular sample & ND & ND & ND & ND & ND & $>32$ & $>32$ & $>32$ & $>32$ & $>32$ & 64 & 16 \\
\hline Rhizopus oryzae Y9 & Sinus biopsy & ND & ND & ND & ND & ND & $>32$ & $>32$ & $>32$ & $>32$ & $>32$ & 128 & 64 \\
\hline Lomentospora prolificans Y8 & Blood & ND & ND & ND & ND & ND & $>32$ & 64 & $>32$ & $>32$ & $>32$ & 32 & 16 \\
\hline Scedosporium apiospermum F2 & Bronchial aspiration & ND & ND & ND & ND & ND & 2 & 0.75 & $>32$ & 4 & ND & 16 & 16 \\
\hline Lichtheimia corymbifera ST87 & Eyes & ND & ND & ND & ND & ND & 2 & 2 & $>32$ & 2 & 2 & 32 & 32 \\
\hline
\end{tabular}

Anid - Anidulafungin; Mica - Micafungin; Casp - Caspofungin; Flu - Fluconazole; 5-Fc - 5-Flurocytosin; Pos - Posaconazol, Itra - Itraconazole; Vori - Voriconazole; AB Amphotericin B; Isavu - Isavuconazole; Ct - Colistin; PMB- Polymyxin B; MIC - Minimum Inhibitory Concentration; ND: Not Done

MacFarland. A 1:100 dilution followed by a 1:20 dilution were performed on yeast strains to obtain a final inoculum of 0.5 to $2.5 \times 10^{3} \mathrm{CFU} / \mathrm{mL}$, whereas only a $1: 50 \mathrm{di}$ lution was done for moulds with a final inoculum of approximately 0.4 to $5 \times 10^{4} \mathrm{CFU} / \mathrm{mL}$. It is important to mention that fresh conidia of filamentous fungi were obtained after approximately 7 days of incubation at $35^{\circ} \mathrm{C}$ on potato dextrose agar. Then, $100 \mu \mathrm{l}$ of the fungal/bacterial inoculum was added into each colistin or PMB-containing wells. Plates were incubated at $37^{\circ} \mathrm{C}$ for $24 \mathrm{~h}$ (Candida spp., bacterial strains) or $48 \mathrm{~h}$ (Cryptococcus) and at $35^{\circ} \mathrm{C}$ for $48 \mathrm{~h}$ (filamentous fungi and Rhodothorula mucilaginosa). The susceptibility to polymyxin antibiotics was assessed on the basis of visual observation of growth or inhibition of the isolate in the culture media. The Resazurin (Sigma Aldrich, St Louis, France) was used to indicate the growth of any microorganism by a culture medium colour shift from blue to pink. Then, the inhibition rate was calculated, after measuring the optical density (OD) value by using a plate reader spectrophotometer (Multiskan spectrum, Thermo Scientific, France), as follows: \% of fungal growth inhibition $=(\mathrm{OD}$ of untreated well $-\mathrm{OD}$ of tested well)*100/(OD of untreated well - OD of blank well); where a blank well contains only the RPMI medium (i.e. without any fungal strain and without any antibiotic agent), an untreated well contains a given strain in the RPMI medium without any antibiotic agents and a tested well contains both a given strain in the RPMI medium and a given antibiotic agent. Thus, the growth inhibition rate is close to $100 \%$ when the concentration of an antibiotic reaches to the MIC as the OD of the tested well is quasi-equal to the OD of the blank well.

\section{Colistin time-kill experiment and minimum fungicidal concentration (MFC) determination}

Colistin time-kill study was performed, as previously described [22]. $9 \mathrm{ml}$ of the fungal suspension was adjusted to a $0.5 \mathrm{McF}$ arland turbidity. One $\mathrm{ml}$ of the adjusted fungal suspension was added to $9 \mathrm{ml}$ of either RPMI-1640 medium, as a control, or to a solution of growth medium supplemented with an appropriate concentration of antibiotic solution. The colistin concentrations in the resulting solutions were $0.5,1,2,4,8,16$, and 32 times the MICs for the tested isolates. Then, the tubes were incubated at $37^{\circ} \mathrm{C}$ on an orbital shaker. At 0 , $6,12,24,36$, and $48 \mathrm{~h}$ following the introduction of the tested isolate into the solutions tubes, $100 \mu \mathrm{l}$ aliquots were taken from each test solution. Different serial dilutions were performed on these aliquots, and a $10 \mu \mathrm{l}$ aliquot from each dilution was streaked on Sabouraud agar plates (Biomérieux, France) and incubated for approximately $24 \mathrm{~h}$ for colony count determination. Then, the Minimum fungicidal concentration (MFC) was determined as the lowest antibiotic concentration leading to no significant growth or less than three colonies on Sabouraud agar plates in comparison to the growth control. The experimental data was analyzed using the GraphPad Prism 5.3 software (GraphPad Inc., San Diego, CA, USA) to obtain time-kill curves. 


\section{Study of the mechanism of action of colistin on Candida} albicans species

We used propidium iodide (PI), a membrane-impermeable DNA stain, to demonstrate the eventual fungicidal activity of colistin by its ability to induce cell membrane damages [23]. Colistin-treated and untreated cells were suspended in PBS and stained with $5 \mu \mathrm{g} / \mathrm{ml}$ of PI for $20 \mathrm{~min}$ in the dark at room temperature. PI fluorescence was examined under a fluorescence microscope at $\lambda_{\mathrm{ex}}=535 \mathrm{~nm}$ and $\lambda_{\mathrm{em}}$ $=617 \mathrm{~nm}$.

\section{Colistin / antifungals association checkerboard testing}

Checkerboard broth microdilution method was used to test the synergy of colistin with three antifungal agents commonly used in clinical settings; amphotericin B, fluconazole and itraconazole (Sigma Aldrich, St Louis, France). Firstly, MICs of individual agents were determined because the range of concentration of drugs to test the associations was established according to these MICs. Eight doubling dilutions of the two agents being tested (i.e. colistin and antifungals) were prepared in the susceptibility testing medium RPMI-1640 (1MIC, $1 / 2$ MIC, $1 / 4 \mathrm{MIC}, 1 / 8 \mathrm{MIC}, 1 / 16 \mathrm{MIC}, 1 / 32 \mathrm{MIC}, 1 / 64 \mathrm{MIC}$, 1/128 MIC). $50 \mu \mathrm{l}$ of each agent was added in wells of a microtiter plate to provide a total of 64 drug combinations. Additional rows were used to determine the MIC of each antimicrobial agent alone by adding $100 \mathrm{ml}$ of each agent. The fungal inoculum was prepared according to the CLSI standard protocol and $100 \mu \mathrm{l}$ was added to each well. The plates were incubated under optimal growth conditions for yeasts and filamentous moulds. The results were analysed and $\mathrm{MIC}_{100}$ were determined visually and by optical density measurements on a microplates reader (Multiskan Spectrum, Thermo Scientific), based on a reduction in absorbance compared to the free drug control wells. Then, we calculated the Fractional Inhibitory Concentrations (FICs), where FIC1 $($ Colistin $)=$ MIC of colistin in the combination $/ \mathrm{MIC}$ of colistin alone and FIC2 (antifungal drug) = MIC of antifungal drug in the combination/MIC of antifungal drug alone. The fractional inhibitory index (FIX) is the sum of FIC1 and FIC2 and was interpreted as follows: if the FIX is $\leq 0.5$, then there is synergy between the tested antimicrobials; if it is $>0.5$ but $\leq 1$ then there is additivity between the tested antimicrobials; if it is $>1$ but $\leq 4$, there is indifference between the tested antimicrobials, and if the FIX is $>4$, that means that there is an antagonism between the tested antimicrobials.

\section{Results}

Resistance phenotypic profiles of the emerging fungal pathogens used in this study

The MICs obtained with the different antifungal classes tested against the 4 Candida spp. and Aspergillus spp. quality control strains corresponded to the recommended $24 \mathrm{~h}$ and $48 \mathrm{~h}$ - MICs limits of microbroth dilution method outlined in the CLSI protocol. This confirms the adequateness of the method used to determine susceptibility profiles for all isolates. C. albicans H6 was resistant to all azole antifungal agents tested while $C$. albicans $\mathrm{H} 5$ was only resistant to fluconazole (Table 1). Both strains remained sensitive to echinocandins and amphotericin B antifungals. Rhodotorula mucilaginosa was resistant in vitro to fluconazole with MIC $=128 \mu \mathrm{g} / \mathrm{ml}$ and to all echinocandin agents. Cryptococcus neoformans was also resistant to echinocandin class (Table 1).

All filamentous fungi tested were resistant to amphotericin B with a high MIC for Rhizopus oryzae and Scedosporium species (>32 $\mu \mathrm{g} / \mathrm{ml}$ ). Fusarium, Scedosporium and Rhizopus species were also resistant to azoles (voriconazole, itraconazole and posaconazole) usually used in clinical settings (Table 1). The pyrimidine analogue 5-fluorocytosine and echinocandin agents were not tested against moulds because of their relatively poor activity against filamentous fungi.

\section{Polymyxins exhibited in vitro, antifungal activity against MDR yeasts and filamentous fungi}

Susceptibility testing showed clear endpoints with a $100 \%$ growth inhibition of the MDR strains tested. The MICs of Escherichia coli LH1 (MIC $=8 \mu \mathrm{g} / \mathrm{ml}$ ) (16), Escherichia coli $1 \mathrm{R} 4(\mathrm{MIC}=8 \mu \mathrm{g} / \mathrm{ml})(17)$ and Klebsiella pneumoniae $853(\mathrm{MIC}=64 \mu \mathrm{g} / \mathrm{ml})$ [20] were within the quality control ranges.

The polymyxins MICs against the fungi strains ranged from 16 to $128 \mu \mathrm{g} / \mathrm{ml}$ (Table 1), except for Aspergillus fumigatus and A. flavus strains, which appeared to be not sensitive to both colistin and PMB, with MICs $\geq 256 \mu \mathrm{g} / \mathrm{ml}$. Among the 11 clinical strains tested, 4 isolates including Lichtheimia corymbifera, Lomentospora prolificans and Scedosporium apiospermum exhibited the highest susceptibility to polymyxin molecules, with MICs ranging from $16 \mu \mathrm{g} / \mathrm{ml}$ to $32 \mu \mathrm{g} / \mathrm{ml}$; in contrast Aspergillus calidoustus exhibited the lowest susceptibility to colistin and PMB, with MICs of $256 \mu \mathrm{g} / \mathrm{ml}$ and $128 \mu \mathrm{g} / \mathrm{ml}$, respectively.

\section{Colistin presents fungicidal activity against clinical MDR yeasts}

The colistin MICs ranged from 16 to $128 \mu \mathrm{g} / \mathrm{ml}$ for the yeast isolates. As shown on Fig. 1, no inhibitory effect was observed at colistin concentrations equal to $0.5 \mathrm{X}$ MICs and the curves were nearly identical to those found in the controls for all species tested. At colistin concentrations equal to $1 \mathrm{X}$ MICs, fungistatic effect was observed (Fig. 1). In contrast, colistin fungicidal activities against $C$. albicans, $C$. neoformans and $R$. mucilaginosa 


\section{Candida albicans $\mathrm{H} 6$}

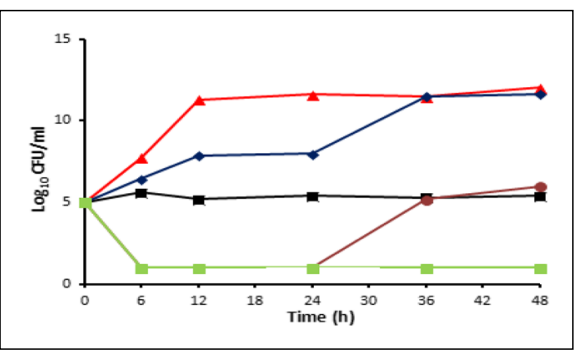

Cryptococcus neoformans

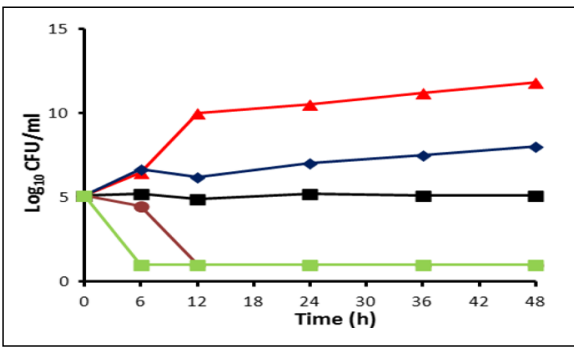

Candida krusei ATCC

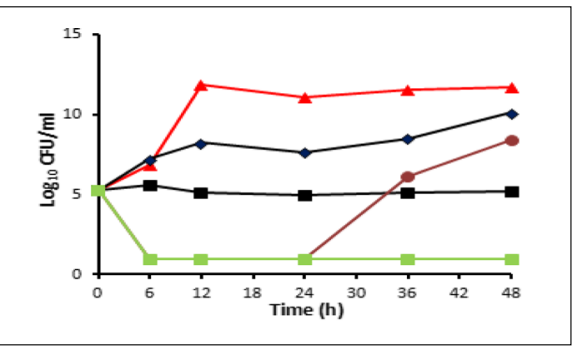

Rhodotorula mucilaginosa H2

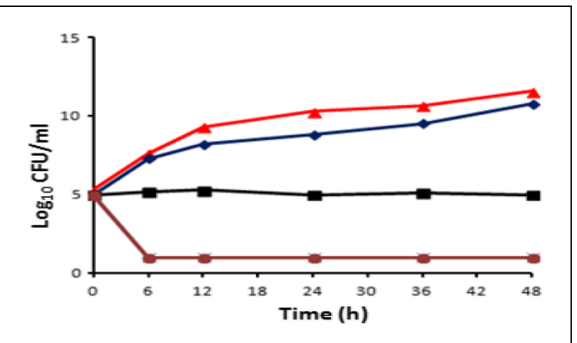

Fig. 1 Time-kill kinetics of colistin against four fungal strains (C. albicans, C. krusei, C. neoformans and R. mucilaginosa). The colistin concentrations

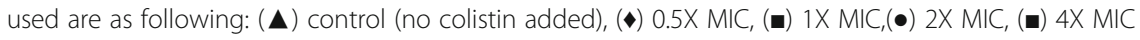

were noted, no later than 12th hour of incubation, with $\mathrm{MFC} \geq$ to two-fold of its MIC values (Fig. 1). For C. albicans strains, the MFC was $256 \mu \mathrm{g} / \mathrm{ml}$, but regrowth was observed at the 36th hour of incubation. The latter phenomenon was not observed in Cryptococcus neoformans or in Rhodotorula mucilaginosa species.

Moreover, a red fluorescence was observed by fluorescence microscopy (Fig. 2) indicating the presence of interaction between PI and nuclear DNA of C. albicans H6 treated with a colistin concentration equal to MFC. This observation allowed us to conclude that colistin can induce cell membrane damage which provide further evidence that it can lead to cell death, confirming its fungicidal activity.
Synergistic activity of colistin with itraconazole, amphotericin B and fluconazole

Based on checkerboard association testing (Fig. 3), colistin-itraconazole and colistin-amphotericin B exhibited a synergistic activity against MDR $C$. albicans and the mucoralean Lichtheimia corymbifera. We noted that itraconazole MIC decreased from $32 \mu \mathrm{g} / \mathrm{ml}$ to $2 \mu \mathrm{g} / \mathrm{ml}$ (for C. albicans H6), with a FIX $=0.5$, and from $2 \mu \mathrm{g} / \mathrm{ml}$ to $0.5 \mu \mathrm{g} / \mathrm{ml}$ (for L. corymbifera), with a FIX $=0.2$ when combined with colistin $(64 \mu \mathrm{g} / \mathrm{ml}$ and $0.5 \mu \mathrm{g} / \mathrm{ml}$, respectively) (Table 2). The MIC of amphotericin B decreased from $2 \mu \mathrm{g} / \mathrm{ml}$ to $0.5 \mu \mathrm{g} / \mathrm{ml}$ (for L. corymbifera) and from $1 \mu \mathrm{g} / \mathrm{ml}$ to $0.5 \mu \mathrm{g} / \mathrm{ml}$ (for C. albicans $\mathrm{H6}$ ) when combined with colistin $(0.5 \mu \mathrm{g} / \mathrm{ml}$ and $1 \mu \mathrm{g} / \mathrm{ml}$, respectively)
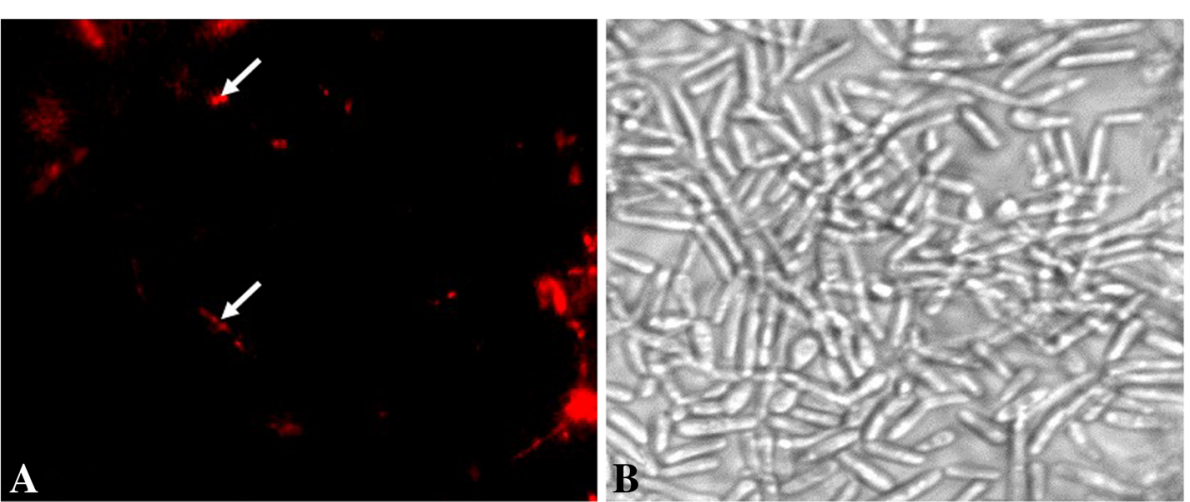

Fig. 2 Fluorescence microscopy of Candida albicans $\mathrm{H} 6$ after treatment with $5 \mathrm{\mu g} / \mathrm{ml}$ of propidium iodide. a: fluorescence image of cells treated with $256 \mu \mathrm{g} / \mathrm{ml}(2 \mathrm{X}$ MIC) of colistin for $24 \mathrm{~h}$. b: Brightfield image of cells treated with colistin for $24 \mathrm{~h}$. Scale bar: $2 \mu \mathrm{m}$ 
A Rhodotorula mucilaginosa

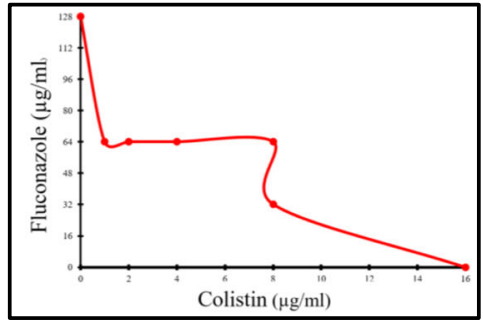

C Lichtheimia corymbifera ST87

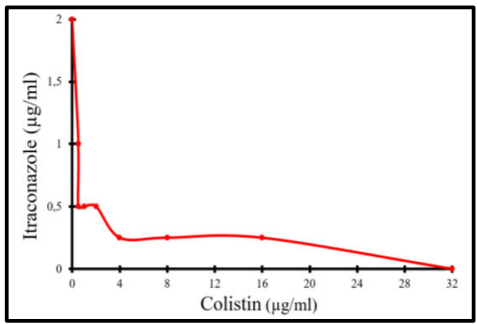

E Lichtheimia corymbifera ST87

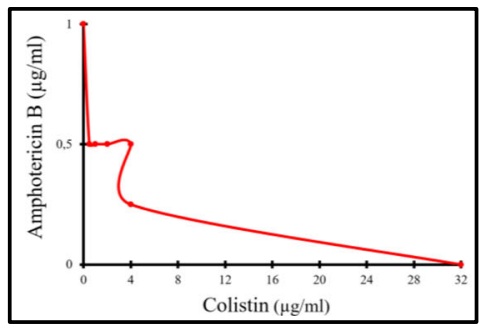

B Candida albicans $\mathrm{H} 5$

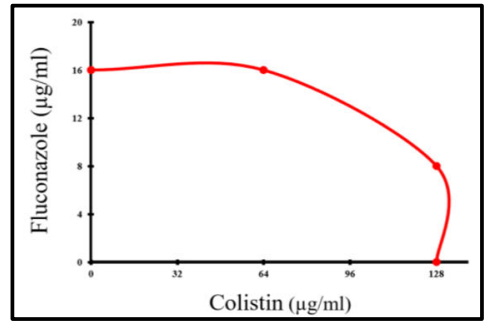

D Candida albicans $\mathrm{H} 6$

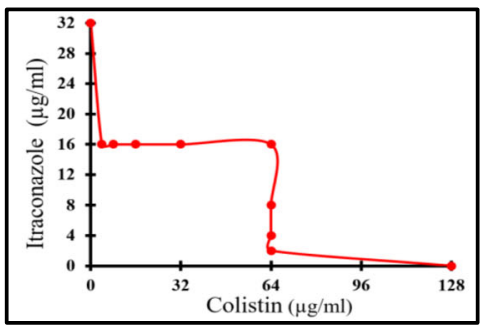

F Candida albicans $\mathrm{H} 6$

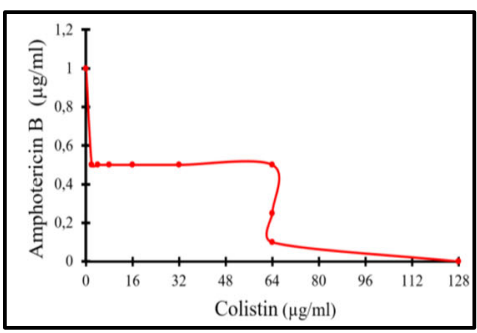

Fig. 3 Plots of the checkerboard assays for the combinations of colistin with 3 antifungals (fluconazole, itraconazole and amphotericin b). Each dot presents the MICs of colistin (x-axis) and the antifungal agent (y-axis) used in the combination against R. mucilaginosa (a), C. albicans H5 (b), L. corymbifera (c and $\mathbf{e})$ and C. albicans $\mathrm{H} 6$ (d and $\mathbf{f}$ )

Table 2 MIC and FIX values of colistin in combination with antifungals from the checkerboard assay

\begin{tabular}{|c|c|c|c|c|c|c|}
\hline Strains tested & Agents in combination & MIC alone $(\mu \mathrm{g} / \mathrm{ml})$ & MIC in the combination $(\mu \mathrm{g} / \mathrm{ml})$ & FIC & FIX & Outcome \\
\hline \multirow[t]{2}{*}{ Rhodotorula mucilaginosa } & Colistin & 16 & 1 & 0.06 & 0.5 & Synergy \\
\hline & Fluconazole & 128 & 64 & 0.5 & & \\
\hline \multirow[t]{2}{*}{ Candida albicans $\mathrm{H} 5$} & Colistin & 128 & 1 & 0.007 & 1 & Additivity \\
\hline & Fluconazole & 16 & 16 & 1 & & \\
\hline \multirow[t]{4}{*}{ Candida albicans $\mathrm{H} 6$} & Colistin & 128 & 64 & 0.5 & 0.5 & Synergy \\
\hline & Itraconazole & 32 & 2 & 0.06 & & \\
\hline & Colistin & 128 & 1 & 0.007 & 0.5 & Synergy \\
\hline & Amphotericin B & 1 & 0.5 & 0.5 & & \\
\hline \multirow[t]{4}{*}{ Lichtheimia corymbifera ST87 } & Colistin & 32 & 0.5 & 0.01 & 0.2 & Synergy \\
\hline & Itraconazole & 2 & 0.5 & 0.25 & & \\
\hline & Colistin & 32 & 0.5 & 0.01 & 0.2 & Synergy \\
\hline & Amphotericin B & 2 & 0.5 & 0.25 & & \\
\hline
\end{tabular}

FIC; Fractional Inhibitory concentration = MIC of the agent in the combination/MIC of the agent alone. FIX; Fractional Inhibitory Index is the sum of the FICs of the agents in the combination 
(Table 2). Interestingly, colistin acted in synergy with fluconazole against $R$. mucilaginosa but not against Candida albicans H5 (Table 2). Although the colistin MICs decreased from $128 \mu \mathrm{g} / \mathrm{ml}$ to $1 \mu \mathrm{g} / \mathrm{ml}$ when combined with fluconazole $(16 \mu \mathrm{g} / \mathrm{ml})$, no synergy against $C$. albicans $\mathrm{H} 5$ was observed with a FIX $=1$ (Table 2).

\section{Discussion}

Our study demonstrated that polymyxin antibiotics have, aside from their antibactericidal activity, an antifungal activity, especially against multidrug-resistant Candida, Rhodotorula and Cryptococcus yeast isolates, but also against resistant filamentous fungi, such as Scedosporium, Rhizopus and Lichtheimia species. The MICs obtained against Candida spp. ranged between 64 and $128 \mu \mathrm{g} / \mathrm{ml}$ for colistin and mainly between 16 and $64 \mu \mathrm{g} /$ $\mathrm{ml}$ for PMB. The latter is in concordance with PMB MICs already reported by Zeidler et al and Zhai et al studies [7, 24] confirming the validity of our results and the reproducibility of the used technique. Although we obtained a MIC of PMB equal to $16 \mu \mathrm{g} / \mathrm{ml}$ against $C$. neoformans, a lower $\mathrm{MIC}(\mathrm{MIC}=8 \mu \mathrm{g} / \mathrm{ml})$ against this species has been previously described [24]. This could possibly be explained by the resistance phenotype of the strain tested in our study. Moreover, colistin has not been shown, in the early 1970s, to be an effective molecule against three strains belonging to the genus Rhodotorula [14]. However, to the best of our knowledge, neither colistin MICs against C. neoformans nor PMB MICs against $R$. mucilaginosa have been reported elsewhere.

On the other hand, the filamentous fungi isolates tested in this study constitute the most common emerging cause of human mould infections with an increase being reported from various geographical sites [2], including particularly Aspergillus and Fusarium spp. Here, colistin and PMB MICs ranging from 16 to $64 \mu \mathrm{g} /$ $\mathrm{ml}$ have been observed against the $F$. oxysporum and $F$. Solani strains which are resistant to almost all azole antifungals and amphotericin B. The same range of MICs has been reported in previous study where 12 Fusarium spp. were tested against $\mathrm{PMB}$ but not against colistin [16]. The absence of colistin and PMB efficacy against several Aspergillus spp. has been reported in various studies with MICs $>256 \mu \mathrm{g} / \mathrm{ml}[24,25]$ which are similar to our results. Despite that Aspergillus spp. are remaining the first cause of mould infections, mucormycosis is increasingly reported in immune-compromised patients and is associated with an elevated rate of mortality (40-70\%) even under an appropriate therapy [26]. Among mucoralean pathogens, Rhizopus is the main frequently identified genus in human infections. In our study, colistin and PMB MICs against $R$. oryzae are one to two folds higher than those reported in earlier studies
$[15,24]$. Indeed, in Ben-Ami et al study, the colistin MICs against the fourteen clinical Rhizopus spp. tested were variables and ranged between 16 and $32 \mu \mathrm{g} / \mathrm{ml}$, but MICs of antifungal agents were not mentioned [15]. So the discordance of MIC results between the Ben-Ami et al study and our work could be explained by the eventual high resistance level of our strain to azole agents which can be due to the over expression of efflux-pumps and/or other mechanisms [27]. It is important to mention that PMB MIC against $R$. oryzae was equal to $64 \mu \mathrm{g} / \mathrm{ml}$ in this study compared to $32 \mu \mathrm{g} /$ $\mathrm{ml}$ obtained by Zhai et al study [24].

Finally, among the pertinent emerging fungal pathogens shown by several studies, Scedosporium and Lomentospora spp. are often notified [28]. They can induce a broad range of diseases; from colonisation in cystic fibrosis patients (for Scedosporium spp.) to disseminated severe infections in immuno-compromised hosts (for Lomentospora prolificans). Although, the colistin MIC against S. apiospermum strain tested here is within the colistin MICs range previously described by Schemuth et al, the colistin MIC obtained for L. prolificans was higher than that described in this previous study $(32 \mu \mathrm{g} / \mathrm{ml}$ versus $12 \mu \mathrm{g} / \mathrm{ml})$ [29]. Nevertheless, it is worthy to note that $\mathrm{MICs}_{90}$ were used by Schemuth et al [29] whereas $\mathrm{MICs}_{100}$ were used in our study. Finally, to the best of our knowledge, colistin and PMB activities against Lichtheimia corymbifera have not been previously reported.

In human studies, a single dose of 75 to $150 \mathrm{mg}$ of colistin produced bioactive serum colistin concentrations ranging from 6 to $18 \mu \mathrm{g} / \mathrm{ml}$; higher serum colistin concentrations ( 13 to $32 \mu \mathrm{g} / \mathrm{ml}$ ) were measured during the prolonged therapy of patients with cystic fibrosis [15]. Therefore, the obtained MICs of colistin and PMB are difficult to be achieved with IV administration, mainly due to their renal and neurological toxicities and the risk of frequent selection of bacterial resistant strains.

However, the efficacy of polymyxin molecules on a large number of MDR fungi can be considered advantageous to treat bacterial and fungal co-infections that occur frequently in immunocompromised patients [30] and cystic fibrosis $(\mathrm{CF})$ patients. Chronic bacterial and fungal colonization of the respiratory tract secretions is the main cause of morbidity and mortality in CF patients. Therefore, it would be helpful to use a treatment that is active on both bacteria and fungi in this context. It is worthy to note that, in clinical practice, colistin is administered by inhalation in CF patients as prophylaxis and also as a treatment against Pseudomonas aeruginosa infection [31]. In addition, aerosolised colistin treatment, is used in ventilator-associated pneumonia (VAP) cases caused by MDR bacteria in intensive care unit setting [32]. Interestingly, in a recent in vivo study, Landersdorfer 
et al [33] observed high epithelial lining fluid and low plasma colistin concentrations following the administration of only a pulmonary dose through jet nebulization, confirming a benefit of the local administration of colistin in comparison to its IV treatment [34]. Moreover, a prospective study conducted on 18 patients with chronic lung disease showed that nebulized colistin is effective and improves the quality of life, without presenting side effects and without selecting colistin-resistant isolates in treated patients [35]. So high-dose nebulized colistin could be proposed against pulmonary life-threatening MDR fungi, without increasing colistin plasma concentration, and thus avoiding colistin's toxicity.

Similar to CF cases, the use of polymyxin antibiotics can improve the poor prognosis of fungal keratitis, due to the emergence of MDR fungal pathogens, particularly Fusarium spp. [36], and to the limited ocular penetration of antifungals [37]. Notably, PMB can be formulated for ophthalmic use [16], which is described as a highly effective drug on bacterial corneal ulcerations [38]. Moreover, the use of such antimicrobial agent constitutes a potential alternative treatment that may improve the outcome in some critical infections caused by MDR fungi, such as the recent MDR Fusarium keratitis-case report in a 46-year-old man who was still declining even the maximal therapeutic support and therapeutic keratoplasty [36].

Several approaches could be used to overcome the development of antifungal resistance in the treatment of fungal diseases. Aside from the discovery of new effective agents, one realistic alternative option would be to enhance the activity of existing agents. Combination therapies exploit the chances for better efficacy, decreased toxicity and reduced development of drug resistance [39]. A previous study demonstrated an in vitro synergy between colistin and echinocandins in several pathogenic yeasts, namely C. albicans, C. glabrata, C. tropicalis, C. parapsilosis and C. krusei, as well as in fluconazole-resistant C. albicans strains [7].

To the best of our knowledge, no previous studies have tested the activity of colistin in combination with other antifungal agents against fungi of the genera Rhodotorula and Lichtheimia.

A high decrease of colistin's MICs was observed when it was combined with azoles (with fluconazole against $R$. mucilaginosa and with itraconazole against either $C$. albicans or L. corymbifera, Table 2 and Fig. 3). It is well known that the main mechanism of action of azoles is the inhibition of enzymes that transform lanosterol into ergosterol, a major lipid of the fungal membrane. This inhibition alters both the permeability and fluidity of fungal membrane [40]. On the other hand, polymyxins are well known for weakening the outer membrane in Gram-negative bacteria and the disruption of its permeability leading to a leakage of intracellular components [41]. Therefore, and as supported by PI staining results (Fig. 2), it is likely that antifungal azoles ease the polymyxins' action and add a potential damage to the fungal membrane which results in a synergistic potency of the combined drugs. Moreover, colistin MIC values significantly decreased from $128 \mu \mathrm{g} / \mathrm{ml}$ to $1 \mu \mathrm{g} / \mathrm{ml}$ and from $32 \mu \mathrm{g} / \mathrm{ml}$ to $0.5 \mu \mathrm{g} / \mathrm{ml}$ against $C$. albicans and $L$. corymbifera respectively when it was associated with amphotericin B. The association of the fungal membrane permeabilization induced by amphotericin B via ion channel formation [42] with the probable membrane damage occurred by colistin could explain the decrease of MICs and the synergistic effect between colistin and amphotericin B.

Thus, despite the elevated MICs of colistin found in our work against multidrug-resistant yeast and moulds, the use of colistin, in combination with other antifungal agents, remains an excellent way to avoid the development of fungal resistance and to decrease the antifungal effective concentration usually used in clinical settings [16, 22].

Colistin is one of many AMPs already used in clinical settings [11]. So, in addition to the colistin-antifungal combination evaluated in this study, other AMPs could further be tested to potentiate the antifungal activity of existing antifungal compounds. For example, Wakabayashi et al, previously described the synergistic effect of lactoferin, a human antimicrobial peptide, with clotrimazole against C. albicans [43]. Moreover, lactoferin induced an important decrease of all azoles' MICs tested against azole-resistant Candida spp. [43]. Consequently, natural or synthetic AMPs, have been identified as an original therapeutic alternative that could be investigated by medical researchers and pharmaceutical companies. Using the same approach which was used herein, another AMP, less toxic than polymyxins such as bacitracin or gramicidin analogues, could be tested as monotherapy or in association with antifungals against MDR fungi.

\section{Conclusion}

Our findings demonstrate that polymyxins display a broad-spectrum activity against common MDR fungi especially those which are difficult to manage in clinical settings. Unfortunately, polymyxins' MICs against these MDR strains are higher than those that could clinically be used in human therapy, thus the use of such high toxicity-associated concentration of polymyxins presents the major limitation of their application in clinical mycology practice. However, colistin seems to induce C. albicans membrane damages and to act in synergy with either itraconazole or amphotericin B (each also acting on the fungal membrane). We therefore suggest that colistin (at a 'safe' reduced dose) can be used in combination with currently available antifungal drugs, as a last resort option, against life-threatening MDR fungi. 


\section{Abbreviations}

AMPs: Antimicrobial peptides; CF: Cystic fibrosis; CLSI: Clinical and Laboratory Standards Institute; FIC: Fractional inhibitory concentration; FIX: Fractional inhibitory index; MDR: Multidrug resistant; MFC: Minimal Fungicidal Concentration; MIC: Minimum inhibitory concentration; PBS: Phosphatebuffered saline; PI: Propidium iodide; PMB: Polymyxin B

\section{Acknowledgements}

The author thanks Magdalen Lardière and CookieTrad for English reviewing.

\section{Funding}

This work was supported by the French Government under the « Investissements d'avenir » (Investments for the Future) program managed by the Agence Nationale de la Recherche (ANR, fr: National Agency for Research), (reference: Méditerranée Infection 10-IAHU-03).

This work was supported by the Région Provence-Alpes-Côte-d'Azur and European funding FEDER PRIMI.

\section{Availability of data and materials}

All data are available in Table 1.

\section{Authors' contributions}

JMR and FB were involved in the conception and design of the study. $\mathrm{HY}$ performed the experiments. HY, SR, JMR and FB analysed and interpreted the data. HY and FB co-wrote the manuscript. All authors have read and approved the final manuscript.

\section{Ethics approval and consent to participate}

Not applicable.

\section{Consent for publication}

Not applicable.

\section{Competing interests}

The authors declare that they have no competing interests.

\section{Publisher's Note}

Springer Nature remains neutral with regard to jurisdictional claims in published maps and institutional affiliations.

\section{Author details}

${ }^{1}$ Aix Marseille Univ, IRD, APHM, MEPHI, IHU Méditerranée Infection, 19-21 boulevard Jean Moulin, 13005 Marseille, France. ${ }^{2}$ Aix Marseille Univ, IRD, APHM, SSA, VITROME, IHU-Méditerranée Infection, 19-21 boulevard Jean Moulin, 13005 Marseille, France.

\section{Received: 14 December 2018 Accepted: 10 April 2019}

\section{Published online: 24 April 2019}

\section{References}

1. Morio F, Jensen RH, Le PP, Arendrup MC. Molecular basis of antifungal drug resistance in yeasts. Int J Antimicrob Agents. 2017;50:599-606.

2. Geddes-Mcalister J, Shapiro RS. New pathogens, new tricks: emerging, drugresistant fungal pathogens and future prospects for antifungal therapeutics. Ann N Y Acad Sci. 2018. https://doi.org/10.1111/nyas.13739.

3. Dannaoui E. Antifungal resistance in Mucorales. Int J Antimicrob Agents. 2017;50:617-21.

4. Alcazar-fuoli L, Mellado E. Current status of antifungal resistance and its impact on clinical practice. Br J Haematol. 2014;166:471-84.

5. Kauffman CA. Fungal infections in older adults. Clin Infect Dis. 2001;33:550-5.

6. Kanafani ZA, Perfect JR. Resistance to antifungal agents: mechanisms and clinical impact. Clin Infect Dis. 2008;46:120-8.

7. Zeidler U, Bougnoux ME, Lupan A, Helynck O, Doyen A, Garcia Z, et al. Synergy of the antibiotic colistin with echinocandin antifungals in candida species. J Antimicrob Chemother. 2013;68:1285-96.

8. Vale Silva LA. Molecular mechanisms of resistance of Candida spp. to membrane-targeting antifungals. In: Coste AT and Vandeputte P, editors. Antifungals: from genomics to resistance and the development of nove agents: Caister Academic Press, Poole, UK; 2015. p. 1-26.
9. Gulshan K, Moye-Rowley WS. Multidrug resistance in fungi. Eukaryot Cell. 2007;6:1933-42.

10. Peyclit L, Baron SA, Yousfi H, Rolain JM. Zidovudine: a salvage therapy for mcr-1 plasmid-mediated colistin-resistant bacterial infections? Int J Antimicrob Agents. 2018;(1):11-3.

11. Bondaryk M, Staniszewska M, Zielińska P, Urbańczyk-Lipkowska Z. Natural antimicrobial peptides as inspiration for Design of a new Generation Antifungal Compounds. J Fungi. 2017;3. https://doi.org/10.3390/jof3030046.

12. Landman D, Georgescu C, Martin DA, Quale J. Polymyxins revisited. Clin Microbiol Rev. 2008;21:449-65.

13. Olaitan A, Morand S, Rolain JM. Mechanisms of polymyxin resistance: acquired and intrinsic resistance in bacteria. Front Microbiol. 2014;5:1-18.

14. Nicholls MWN. Polymyxin sensitivity of Candida tropicalis. J Med Microbiol. 1970;3:529-38.

15. Ben-Ami R, Lewis RE, Tarrand J, Leventakos K, Kontoyiannis DP. Antifungal activity of colistin against Mucorales species in vitro and in a murine model of Rhizopus oryzae pulmonary infection. Antimicrob Agents Chemother. 2010;54:484-90.

16. Hsu LH, Wang HF, Sun PL, Hu FR, Chen YL. The antibiotic polymyxin B exhibits novel antifungal activity against Fusarium species. Int J Antimicrob Agents. 2017:49:740-8.

17. Cassagne C, Normand AC, L'Ollivier C, Ranque S, Piarroux R. Performance of MALDI-TOF MS platforms for fungal identification. Mycoses. 2016;59:678-90.

18. Olaitan A, Chabou S, Okdah L, Morand S, Rolain JM. Dissemination of the mcr-1 colistin resistance gene. Lancet Infect Dis. 2016;16:147.

19. Leangapichart T, Gautret P, Brouqui P, Memish ZA, Raoult D, Rolain JM. Acquisition of $\mathrm{mcr}-1$ plasmid-mediated colistin resistance in Escherichia coli and Klebsiella pneumoniae during hajj 2013 and 2014. Antimicrob Agents Chemother. 2016;60:6998-9.

20. Lalaoui R, Bakour S, Livnat K, Assous MV, Diene SM, Rolain JM. Spread of Carbapenem and Colistin-resistant Klebsiella pneumoniae ST512 clinical isolates in Israel: a cause for vigilance. Microb Drug Resist. 2018. https://doi. org/10.1089/mdr.2018.0014.

21. Espinel-Ingroff A, Arthington-Skaggs B, lqbal N, Ellis D, Pfaller MA, Messer S, et al. Multicenter evaluation of a new disk agar diffusion method for susceptibility testing of filamentous fungi with voriconazole, posaconazole, itraconazole, amphotericin B, and caspofungin. J Clin Microbiol. 2007;45: 1811-20.

22. Pankey G, Ashcraft D, Kahn H, Ismail A. Time-kill assay and etest evaluation for synergy with polymyxin B and fluconazole against Candida glabrata. Antimicrob Agents Chemother. 2014;58:5795-800.

23. Kwolek-Mirek M, Zadrag-Tecza R. Comparison of methods used for assessing the viability and vitality of yeast cells. FEMS Yeast Res. 2014;14:1068-79.

24. Zhai B, Zhou H, Yang L, Zhang J, Jung K, Giam CZ, et al. Polymyxin B, in combination with fluconazole, exerts a potent fungicidal effect. J Antimicrob Chemother. 2010;65:931-8.

25. Short G, Rennison C, Gould K, Fisher A. Activity of Colistin against filamentous Fungi isolated from lung transplant recipients. J Hear Lung Transplant. 2010;29:S185-6.

26. Buchanan WL, Schaufele RL, Knudsen TA, Sein T, Zaoutis TE, Chiou CC, et al. Epidemiology and outcome of Zygomycosis: a review of 929 reported cases. Clin Infect Dis. 2005;41:634-53.

27. Pfaller MA. Antifungal drug resistance : mechanisms, epidemiology, and consequences for treatment. Am J Med. 2012;125:S3-13.

28. Pellon A, Ramirez-Garcia A, Buldain I, Antoran A, Martin-Souto L, Rementeria A, et al. Pathobiology of Lomentospora prolificans: could this species serve as a model of primary antifungal resistance? Int J Antimicrob Agents. 2018;51:10-5.

29. Schemuth $H$, Dittmer S, Lackner M, Sedlacek L, Hamprecht A, Steinmann E, et al. In vitro activity of colistin as single agent and in combination with antifungals against filamentous fungi occurring in patients with cystic fibrosis. Mycoses. 2013;56:297-303.

30. Udeani TKC, Moses J, Uzoechina A, Okwori AEJ, Okwosa CN. Microbial aetiologic agents associated with pneumonia in immunocompromised hosts. African J Infect Dis. 2010;4:1-6.

31. Elborn JS, Hodson M, Bertram C. Implementation of European standards of care for cystic fibrosis - control and treatment of infection. J Cyst Fibros. 2009;8:211-7.

32. Abdellatif S, Trifi A, Daly F, Mahjoub K, Nasri R, Ben Lakhal S. Efficacy and toxicity of aerosolised colistin in ventilator-associated pneumonia: a prospective, randomised trial. Ann Intensive Care Springer Paris. 2016;6:1-11. 
33. Landersdorfer Cornelia B, Nguyen T-H, Linh Thuy Lieu GN, Bischof RJ, Meeusen EN, Li J, Nation RL, et al. Substantial targeting advantage achieved by pulmonary Administration of Colistin Methanesulfonate in a large. Antimicrob Agents Chemother. 2017;61:1-15.

34. Carillo C, Pecoraro Y, Anile M, Poggi C, Oliva A, Amore D, et al. Colistinbased treatment of multidrug-resistant gram-negative bacterial pulmonary infections after lung transplantation. Transplant Proc. 2019;51:202-5.

35. Steinfort DP, Steinfort C. Effect of long-term nebulized colistin on lung function and quality of life in patients with chronic bronchial sepsis. Intern Med J. 2007:37:495-8.

36. Sara S, Sharpe K, Morris S. Multidrug-resistant Fusarium keratitis: diagnosis and treatment considerations. BMJ Case Rep. 2016;3. https://doi.org/10.1136/bcr-2016-215401

37. Maharana P, Sharma N, Nagpal R, Jhanji V, Das S, Vajpayee R. Recent advances in diagnosis and management of mycotic keratitis. Indian J Ophthalmol. 2016;64:346.

38. Bosscha MI, Van Dissel JT, Kuijper EJ, Swart W, Jager MJ. The efficacy and safety of topical polymyxin B, neomycin and gramicidin for treatment of presumed bacterial corneal ulceration. Br J Ophthalmol. 2004;88:25-8.

39. Foucquier J, Guedj M. Analysis of drug combinations: current methodological landscape. Pharmacol Res Perspect. 2015;3:1-11.

40. François IEJA, Cammue BPA, Borgers M, Ausma J, Dispersyn GD, Thevissen K. Azoles: mode of antifungal action and resistance development. Effect of miconazole on endogenous reactive oxygen species production in Candida albicans. Antiinfect Agents Med Chem. 2006;5:3-13.

41. Trimble MJ, Mlynarcik P, Kolar M, Hancock REW. Polymyxin: alternative mechanisms of action. Cold Spring Harb Perspect Med. 2016;6:1-22.

42. Gray KC, Palacios DS, Dailey I, Endo MM, Uno BE, Wilcock BC, et al. Amphotericin primarily kills yeast by simply binding ergosterol. Proc Nat Acad Sci. 2012;109:2234-9.

43. Wakabayashi H, Abe S, Teraguchi S, Hayasawa H, Yamaguchi H. Inhibition of hyphal growth of azole-resistant strains of Candida albicans by triazole antifungal agents in the presence of lactoferrin- related compounds. Antimicrob Agents Chemother. 1998;42:1587-91.

Ready to submit your research? Choose BMC and benefit from:

- fast, convenient online submission

- thorough peer review by experienced researchers in your field

- rapid publication on acceptance

- support for research data, including large and complex data types

- gold Open Access which fosters wider collaboration and increased citations

- maximum visibility for your research: over $100 \mathrm{M}$ website views per year

At $\mathrm{BMC}$, research is always in progress.

Learn more biomedcentral.com/submissions 